\title{
CHARACTERIZATION OF FAIRNESS OF INFORMATION DISTRIBUTION IN A MOBILE AGENT BASED ADAPTIVE INFORMATION SYSTEM
}

\author{
IFTIKHAR AHMED AND JAFAR SADIQ \\ Department of Electrical \& Computer Engineering, International Islamic University Malaysia, \\ 53100 Kuala Lumpur, Malaysia \\ e-mail:a.iftikhar@iiu.edu.my,jsadiq@iiu.edu.my
}

\begin{abstract}
The information age is now passing through an evolutionary phase. As the capacity of networks is increasing to handle and process huge volume of information traffic, the management of such information systems is becoming a trivial issue. The Internet being the largest source of information provision is heterogeneous in nature with constantly evolving environment. New information services are added whereas some are modified or removed. User's demands and interests also change constantly. In such a dynamic environment it is very difficult to maintain a coherent picture of the information services. A novel technique proposed recently employs autonomous mobile agents in faded information field architecture to facilitate information provision and servicing in this new era of information age. The architecture is decentralized in nature whereby both the service provider and information seeker communicate through mobile agents. However, the degree of fairness of information distribution and access is still a problem to be addressed. This paper investigates the issue of fairness among information users for access to information in a networked environment that uses mobile agent technology and faded information field architecture to service the system. A number of algorithms were simulated and their effectiveness is discussed in the paper. Simulation results are presented that show a lot of potential to address the issue of fairness in information systems.
\end{abstract}

Keywords: Mobile agents, information systems, intelligent systems.

\section{INTRODUCTION}

Information in today's business environment has become the prime ingredient of a successful business enterprise. The rapid advances in Information and Communication Technologies (ICT) have given new impetus to the way business is conducted on the internet with information being the key element of a successful business enterprise. The traditional paradigms of business, learning and other essential services are undergoing an evolution. The internet is a huge data repository that is expanding without a central authority. The number of worldwide internet users is predicted to exceed one billion by the end of 2005 [1]. Moreover 300 terabytes of information is published online every year [2]. 
As the performance demands of the internet and its usage increase exponentially, the emphasis is shifting from platform centric computing to network centric computing. Information systems have to be designed that are distributed, dynamic and have high assurance and fault tolerance to meet the heterogeneous demands of users.

As ICT advances, the dynamics of e-commerce tend to be more data intensive and complex. The traditional business to customer paradigm is encompassing the business to business transactions as well. Companies have to comprehend the trends and demands of the users quickly in order to survive in the competitive environment of today. The expectations of users and customers have soared high and they seek the provision of a flawless any time any where service.

The traditional information services based on the client-server model therefore cannot cope with the rising demand placed by the complexities of data intensive heterogeneous computing. It is therefore imperative to employ new techniques to address these problems in order to reduce the network traffic and improve user's access time to services. Information fading technique was reported recently which is based on demand-oriented service architecture [3]. The architecture balances the cost of information provision and utilization on the network by employing push and pull mobile agents to service user requests and conduct the business of information provision on the network. In the faded information field (FIF) the service provider distributes the most demanded and popular information contents closer to its vicinity on various nodes. The volume of information is inversely proportional to the distance of information storing node from the service provider. Thus the FIF permits the fairness of information distribution to unspecified users with equal access time.

\section{INFORMATION SERVICE SYSTEM PERFORMANCE REQUIREMENTS}

The wide area network services are gradually creeping into our daily lives with the number of users of these services constantly on the rise. The information systems now involve a constantly changing environment where stringent performance demands are placed on the network. The businesses are taking advantage of the wide area network facility to offer bargains and put various items on sales and special promotions resulting in the users having much more flexibility and choice available making the process of information provision and utilization a complex matter to deal with on the network.

It is thus imperative for an information system to respond to both user needs and service provider (SP) requirements to respond in time to these needs in a rapidly changing environment. The main desirable attributes of such a system therefore should be flexibility, reliability and quick reaction time. Only a system with these properties can successfully satisfy both users and SPs in the current setting of information age.

The traditional data retrieval methodologies on the optimization of digging in a huge data base to satisfy a search request. However, the FIF employs the concept of autonomous provision and utilization of information based on mobile agents. The amount of information to be faded away from SP is a function of network conditions like 
congestion, popularity of information contents and any other criteria programmed into mobile agents [3].

\subsection{The FIF Architecture}

The information in a FIF is distributed on various nodes in the system. The information pattern in the filed is analogous to the electromagnetic radiation radiated from an antenna, high in intensity near the antenna and low in intensity away from the antenna. The SP fades away the information contents to the nodes in the field with the richness in information contents inversely proportional to the distance of the node from the SP through Push mobile agents (MAs). The user on the other hand sends out Pull MAs seeking the desired information [3-4]. The general schematic of FIF architecture is depicted in Fig. 1.

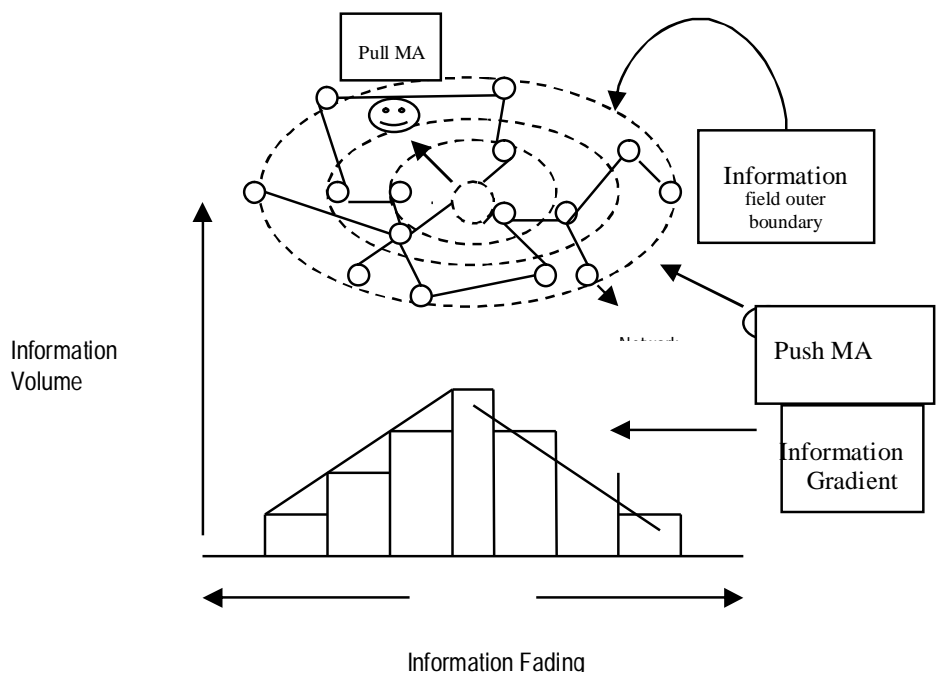

Fig. 1: The layout of FIF architecture.

\subsection{FIF System Components}

The system essentially consists of logically connected nodes through which users and service providers correspond. Mobile agents are used by both parties to acquire and provide information respectively, under evolving/changing situation. The mobile agents (MA) generated by service providers are termed as Push mobile agents (Push MAs). Push MAs carry out the function of autonomous coordination and negotiation with other nodes for information fading according to network situation and the level of importance attached to the information.

The level of importance of particular information content is based on its popularity, determined from a high hit rate of query by the information seeker on the network. The Pull mobile agents (Pull MAs) are generated by users and they autonomously navigate in search of the required information on the network nodes in a step by step fashion. Once the required information is located, these agents report back to the source. The Push and pull MAs have no direct correspondence with each other. 
The third important subsystem of a FIF is the node itself. It is a platform for both storage of information and program execution. It monitors the local information-based system conditions and autonomously makes decisions for allocation requests by the SP. It determines the upper directions to the information service in addition to response times on its upper directions. The upper directions or stages of information eventually point to the SP node whereas the lower directions to the information service lead to the outer nodes away from the SP. Each subsystem is autonomous in terms of control to execute its operations and coordination with other nodes under evolving network conditions.

\subsection{Communication Format in the FIF}

The conventional communications techniques cannot cope with the evolving conditions in a heterogeneous network environment where the state of nodes, the status of the SPs, and the stability of connections are highly unpredictable as the user demand to access the information is dynamic in nature. FIF therefore employs a different technique referred to as content Code communication technique [3]. An example of content code communication is depicted in Fig.3 where the information about a university is structured according to the degree of importance. SP1 specifies the university name followed by CM1 indicating the location of university with respect to country and city. CM2 depicts the programs offered by the university. The message format components thus lead to the breakdown of the principal source of information available on the SP into its uniquely defined characteristic codes (CMs). Push MAs are sent out in the FIF by SPs specifying Content Codes (CMs) of information to the nodes using the message format shown in Fig. 3. The selection of information storage/allocation is autonomously carried out by the nodes based on CMs. Similarly the Pull MAs sent out by the users search for the required information based on CMs.

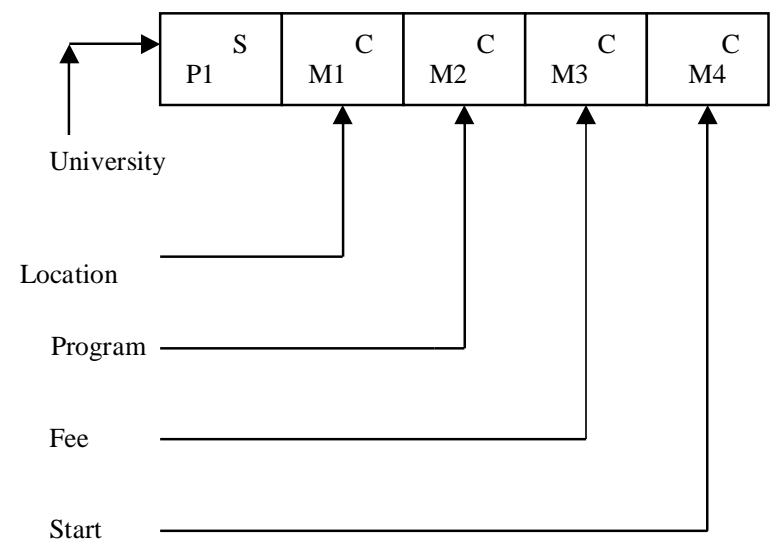

Fig. 2: The message format in a FIF architecture. 


\section{A SURVEY OF MOBILE AGENTS}

A distributed environment is most suitable for the employment of mobile agents. The details of such applications in a distributed environment can be found elsewhere [5-7]. Only, a brief introduction of mobile agents and their role in a network based information system will be discussed. The term mobile agent is often context-dependent and has two separate and distinct concepts: mobility and agency. The term agency implies having the same characteristics as that of an agent. These are self-contained and identifiable computer programs that can move within the network from node to node and act on behalf of a user or other entity. These can halt execution from a host without human interruption [8].

The current distributed network environment is based on the traditional client-server paradigm. In case of mobile agents employed in a network, the service provision and utilization can be distributed in nature and is dynamically configured according to the changing network performance metrics like congestion and user demand for service provision [3]. The two network environments are depicted in Fig. 3 both for client-server and mobile agents.

Mobile agents are typically suited to applications requiring structuring and coordinating wide area network and distributed services involving a large number of remote real time interactions [9].

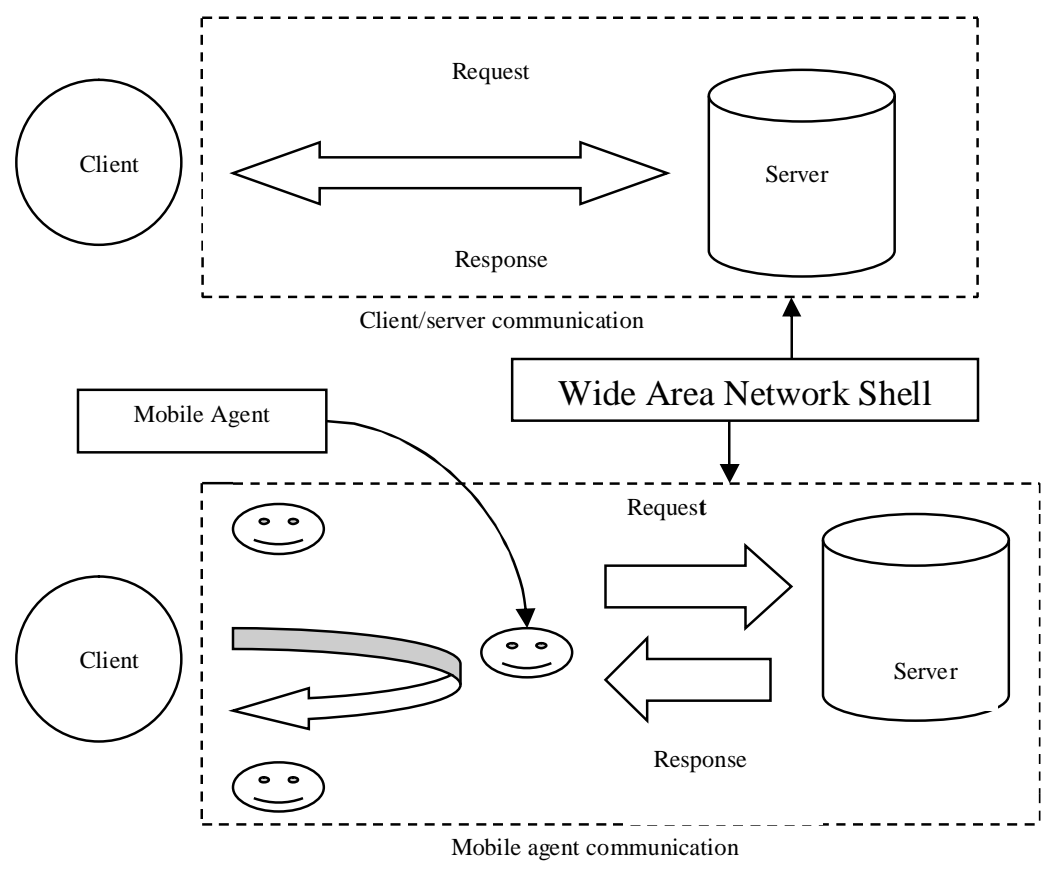

Fig. 3: A mobile agent is a network aware program that can conserve bandwidth in a complex adaptive information system environment. 


\section{FAIRNESS OF INFORMATION DISTRIBUTION AND UTILIZATION SIMULATION}

The purpose of Information fading in a network is twofold:

- Congestion Reduction - The server hosting a popular site is highly likely to get congested. The information provider could employ additional machines (child nodes) to which the original server would distribute or fade its information contents. A Pull MA entering the network of the parent server could then access any of the child nodes, (may be referred to as an SP cluster) which would service the agent's request for the desired information and therefore reduce the service request traffic load on the parent server (SP) in the network.

- Fairness of Access - Service providers (whether commercial or non-commercial) would like their information contents to be easily accessible by the users on the network. The established and renowned service providers stand out compared to new competitors as their search details are already indexed by search engines. Not only the new service providers suffer a long delay in getting their nomenclature indexed by a search engine, but they are the tail enders on the search engine index. This particular scheme of indexing by search engine creates disparity among service providers on the network. A faded information field system therefore can aid in creating fairness among various contenders of information provision and utilization on the network.

For congestion reduction, the service provider must use its own resources to create the field because the congestion reduction benefits only the provider. However, for advertising, each information provider cannot be expected to have the resources to create a field for themselves. In such a scenario, it is possible that each server participates in the faded information field as being part of the field in addition to being a source of information. Thus, no one is the owner of the faded information field resources, and there is neither prohibition nor delay in becoming a member of the field.

For the sake of localization (prioritizing a nearby service provider over a distant one), information should be faded in a geographic area as close as possible around the server. The faded information distance away from the SP was approximated in the simulations using propagation delays of information on the network.

For the sake of fairness, the field size of all SPs should be equal. This is taken to be the number of nodes that constitute the field. Several possible algorithms exist for creating similar field sizes, these are outlined below:

- Sorting. The service provider maintains a list of nodes sorted by delay (as a substitute for physical distance). It can thus determine how "far" it needs to send its Push-MAs to create the standard field size, and imposes a travel restriction beyond the envisaged perimeter of its field. The Push-MAs will be required to keep updating the field until they reach the boundary of the SPs field. Alternatively the Push-MAs can be multicast to the required nodes in the field. Sorting technique is resource intensive since it must be employed to account for cost update in the system as and when it occurs. 
- Step Size. The service provider determines the average step distance (D) from each server from the closest to the furthest. The approximate distance that the SP envisages to cover in its FIF is given by the relation

$$
\mathrm{S}=\mathrm{D} \times \mathrm{N}
$$

where $\mathrm{N}$ is the number of nodes on the network constituting the FIF of the SP where the information contents will be distributed. The step size must be recomputed every time there is an update in the system. During the course of simulation, it was discovered that for 50 servers, this procedure was twice as fast as the sorting strategy discussed earlier.

- Averaged Step Size. In order to account for skewing of SPs' distances, an average distance is computed for each server that is used to determine the step size instead. For a total of 50 servers simulated, its time to execute was not significantly different from the Step Size algorithm.

- Modified Averaged Step Size. The averaged step size algorithm still gets affected by the skewing of data. The algorithm was modified by computing the average step size of all the costs excluding the minimum and maximum costs of system information updates.

A simulation engine was programmed that mimicked a wide area network environment. The performance of each of the aforementioned algorithms was tested by simulation using the programmed simulation engine with randomly distributed servers connected to randomly distributed gateways. The simulation involved 50 servers and 200 routers/gateways. Djikstra's algorithm [10] was used to determine the server-to-server costs. Under a standard policy used in the simulations, each server was allowed to maintain a field size of 10 nodes. Figure 4 demonstrates the field size distribution for this wide area network.

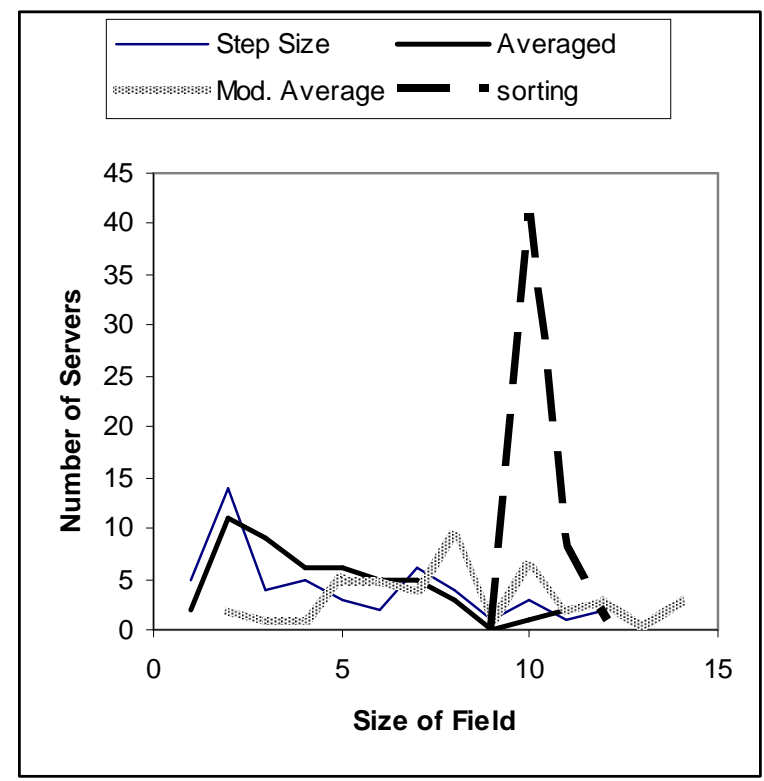

Fig. 4: Field size distribution versus the number of nodes in the network. 
It was observed that the sorting algorithm gave the closest approximation to the required distribution. However, the step size method failed to give the required distribution when it was required to provide a field size of 10 nodes. The averaged step size method faired a little better. It gave better field distribution since the distances are skewed to the higher end thus diminishing the field size below the required size. However, the modified average method gave a very good average of 10.8 nodes per faded information field but the servers did not all have equal numbers of nodes in their fields in this case.

In order to account for skewing, a multiplier was introduced into the algorithms for step size and averaged step size. The step size algorithm was set to produce a field size of 15 nodes and the averaged step size algorithm a field size of 16 nodes. The results are shown in Fig. 5.

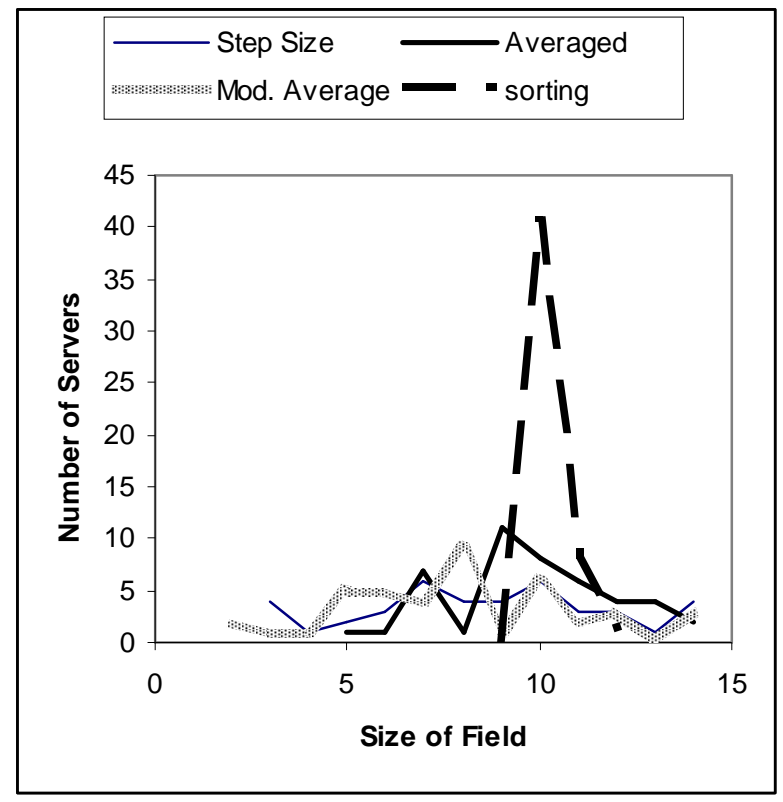

Fig. 5: Compensation for skewing.

It can be seen that the field sizes are better distributed with no processing overhead. The averaged step size results finally show a significant improvement over the normal step size results, as expected. In the second simulation run, the sorting algorithm had an average field size of 10.2 nodes per information provider, the step size algorithm had 10.3, the averaged step size had 10.5 and the modified average had 10.8 nodes respectively. Also, the averaged step size algorithm gave a fair distribution of field size, with most of the servers within a certain range.

The simulation was repeated using 150 routers instead of 200 to determine the effect of network size on the field size. The number of servers was kept the same. Figure 6 shows the field size without any multiplier and Fig. 7 depicts the field size with a multiplier. Again, the sorting and modified average algorithms both produced the required field size, whereas the other two algorithms did not. The step size algorithm this time needed only a multiplier of 1.2 to give an acceptable average, but the average step size algorithm still 
needed a multiplier of 1.6. The latter produced a decent distribution of field size. and further simulation is required to determine the effects of network size on the above algorithms, as well as the effect of required field size. The preliminary simulations demonstrated that the sorting algorithm gives the best distribution of field size. Without a multiplier, the modified average algorithm gives the best performance, whereas, with a multiplier, the averaged step size algorithm gives the best distribution among the three non-sorting algorithms.

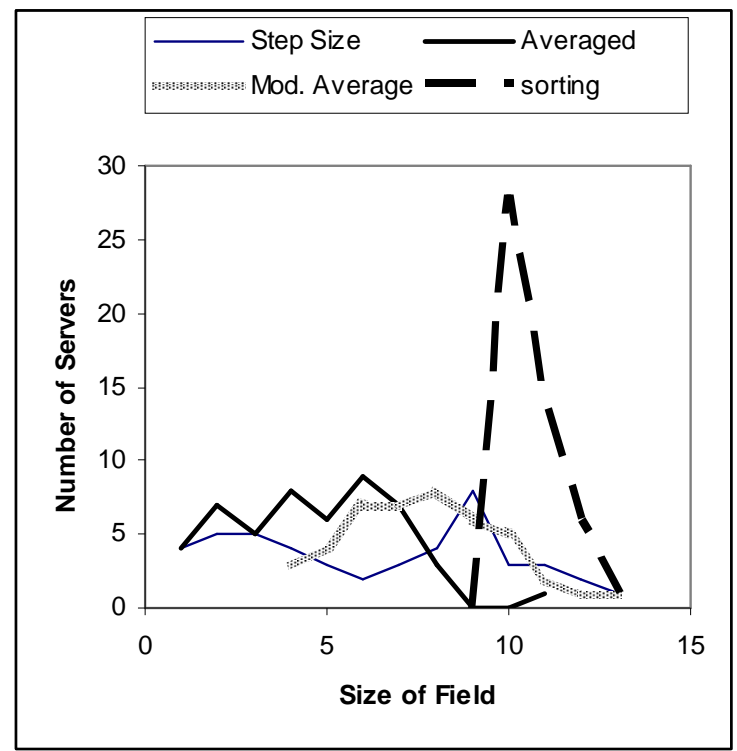

Fig. 6: Field size without a multiplier.

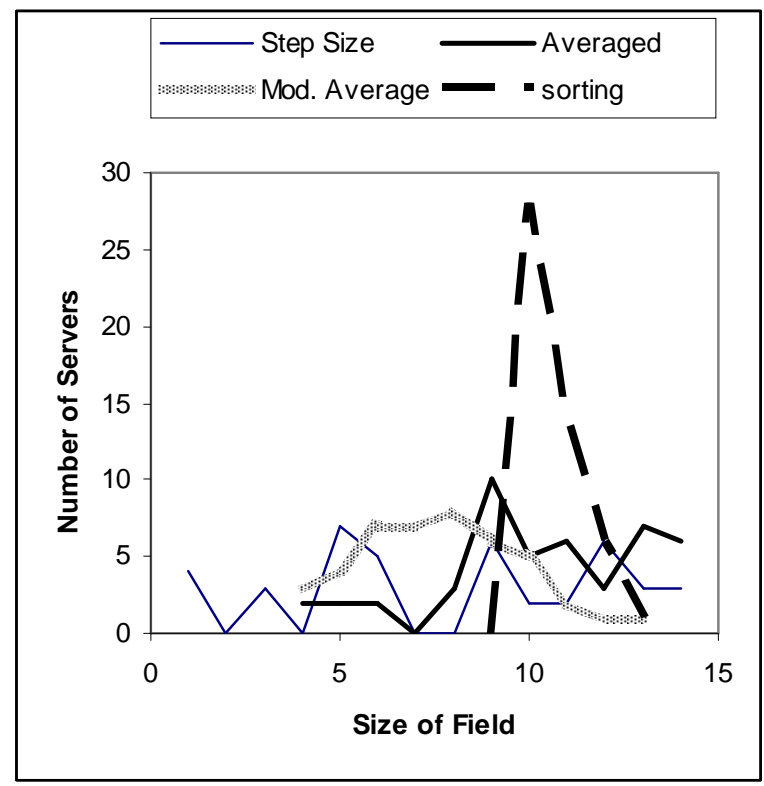

Fig. 7: Field size with a multiplier. 


\section{CONCLUSION}

Faded information filed concept has been reviewed in addition to a survey of mobile agents. A faded information field was simulated by programming a network simulation engine. A number of algorithms were tested on the simulation engine to characterize the network behavior with respect to fairness of information distribution by various service providers on the network. The simulation provided a comparative analysis of algorithms to achieve this goal in addition to discovering a need to introduce a multiplicative factor to fine tune the performance of the algorithms evaluated for the purpose. It was discovered that without a multiplier, the modified average algorithm gave the best performance. But with a multiplier, the averaged step size algorithm turned out to be the best in distributing the information fairly distribution among the three non-sorting algorithms. These results indicate that the faded information technique can be used to provide equal access rights to the information seekers and equal information distribution rights to the service providers on the network thus creating fairness of information provision and utilization.

\section{REFERENCES}

[1] L. G. Roberts," Beyond Moore's Law: Internet Growth Trends," IEEE Computer, Vol. 33, no. 1, p.117, 2000.

[2] P. Lyman and H. R. Varian, "How much Information" Journal of Electronics Publishing, Vol. 6, Issue 2, December 2000.

[3] H. F. Ahmad, H. Arfaoui and K. Mori, "Autonomous Information Fading by Mobile Agents for improving User's Access Time and Fault Tolerance," Proceedings, 5th International Symposium on Autonomous Decentralized Systems, p. 279-283, 2001.

[4] H. Arafoui and K. Mori," Autonomous Navigation Architecture for Load Balancing User demands in Distributed Information Systems," IEICE Transactions on Communications, E84B(10), p.1085-1093, 2001.

[5] A. Karmouch and V. A. Pham, "Mobile Software Agents: An Overview," IEEE Communications Magazine, Vol. 36, No. 7, July 1998.

[6] M. K. Perdikeas et al, "Mobile Agents Standards and Available Platforms," Comp.Net., Vol. 31, No. 19, 1999, p. 1999-2016, August 1999.

[7] D. B. Lange and M. Oshima, "Seven Good Reasons for Mobile Agents," Communications of the ACM, Vol. 42, No. 3, March 1999, p. 88-89, March 1999.

[8] J. Cao, G. H. Chan, W. Jia, and T. Dillon, "Check-pointing and Rollback of wide-area Distributed Applications using Mobile Agents," Proceedings International Parallel and Distributed Processing Symposium. San Francisco: IEEE Computer Society Press, April 2001.

[9] S. Funfrocken," Integrating Java-based Mobile Agents into Web Servers under Security Concerns," Proceedings $31^{\text {st }}$ Hawaii International Conference on System Sciences, p.34-43, January. 1998.

[10] Marta Steenstrup," Routing in Communications Network," Prentice Hall New Jersey, USA, p.143-144, 1995. 